\title{
GEN-O-MA project: an Italian network studying clinical course and pathogenic pathways of moyamoya disease-study protocol and preliminary results
}

\author{
Anna Bersano ${ }^{1}$ (1) $\cdot$ Gloria Bedini $^{2} \cdot$ Sara Nava $^{2} \cdot$ Francesco Acerbi $^{3} \cdot$ Davide Rossi Sebastiano $^{4} \cdot$ Simona Binelli $^{4}$. \\ Silvana Franceschetti ${ }^{4}$. Giuseppe Faragò ${ }^{5}$ - Marina Grisoli ${ }^{6}$. Andrea Gioppo ${ }^{5}$ - Paolo Ferroli ${ }^{3}$. \\ Maria Grazia Bruzzone ${ }^{6}$. Daria Riva ${ }^{7}$. Elisa Ciceri ${ }^{6}$. Chiara Pantaleoni $^{7}$ - Veronica Saletti ${ }^{7} \cdot$ Silvia Esposito $^{7}$. \\ Nardo Nardocci ${ }^{8}$. Federica Zibordi ${ }^{8}$. Luigi Caputi ${ }^{1}$. Stefania Bianchi Marzoli ${ }^{9}$. Maria Luisa Zedde ${ }^{10}$. \\ Marco Pavanello ${ }^{11}$. Alessandro Raso ${ }^{11}$ - Valeria Capra ${ }^{11} \cdot$ Leonardo Pantoni $^{12}$. Cristina Sarti $^{13} \cdot$ Alessandro Pezzini $^{14}$. \\ Filomena Caria ${ }^{14}$. Maria Luisa Dell' Acqua ${ }^{15}$. Andrea Zini ${ }^{15}$. Claudio Baracchini ${ }^{16}$. Filippo Farina ${ }^{16}$. \\ Sandro Sanguigni ${ }^{17}$. Maria Luisa De Lodovici ${ }^{18}$. Giorgio Bono ${ }^{18}$. Fioravanti Capone ${ }^{19} \cdot$ Vincenzo Di Lazzaro $^{19}$. \\ Silvia Lanfranconi ${ }^{20}$. Massimiliano Toscano ${ }^{21}$ • Vittorio Di Piero ${ }^{21}$ - Simona Sacco ${ }^{22}$. Antonio Carolei ${ }^{22}$. \\ Danilo Toni $^{21} \cdot$ Maurizio Paciaroni ${ }^{23} \cdot$ Valeria Caso ${ }^{23}$. Patrizia Perrone ${ }^{24}$. Maria Vittoria Calloni ${ }^{24} \cdot$ Alfredo Romani $^{25}$. \\ Marco Cenzato ${ }^{26}$. Alessia Fratianni ${ }^{26}$. Emilio Ciusani ${ }^{27}$. Paolo Prontera ${ }^{28}$. Elisabeth Tournier Lasserve ${ }^{29}$. \\ Kinga Blecharz ${ }^{30}$. Peter Vajkoczy ${ }^{30}$ - Eugenio Agostino Parati ${ }^{1}$ • on behalf of GEN-O-MA study group
}

Received: 27 February 2018 / Accepted: 28 November 2018

(C) Fondazione Società Italiana di Neurologia 2019

\begin{abstract}
Background GENetics of mOyaMoyA (GEN-O-MA) project is a multicenter observational study implemented in Italy aimed at creating a network of centers involved in moyamoya angiopathy (MA) care and research and at collecting a large series and biorepository of MA patients, finally aimed at describing the disease phenotype and clinical course as well as at identifying biological or cellular markers for disease progression. The present paper resumes the most important study methodological issues and preliminary results.

Methods Nineteen centers are participating to the study. Patients with both bilateral and unilateral radiologically defined MA are included in the study. For each patient, detailed demographic and clinical as well as neuroimaging data are being collected. When available, biological samples (blood, DNA, CSF, middle cerebral artery samples) are being also collected for biological and cellular studies.

Results Ninety-eight patients (age of onset mean \pm SD $35.5 \pm 19.6$ years; $68.4 \%$ females) have been collected so far. $65.3 \%$ of patients presented ischemic (50\%) and haemorrhagic (15.3\%) stroke. A higher female predominance concomitantly with a similar age of onset and clinical features to what was reported in previous studies on Western patients has been confirmed.

Conclusion An accurate and detailed clinical and neuroimaging classification represents the best strategy to provide the characterization of the disease phenotype and clinical course. The collection of a large number of biological samples will permit the identification of biological markers and genetic factors associated with the disease susceptibility in Italy.
\end{abstract}

Keywords Moyamoya disease $\cdot$ Network $\cdot$ Markers $\cdot$ Endothelial progenitor cells $\cdot$ Genetics

Anna Bersano

anna.bersano@gmail.com

Extended author information available on the last page of the article

\section{Introduction}

Moyamoya angiopathy (MA) is a chronic cerebrovascular condition characterized by a progressive stenosis of the terminal part of the supraclinoid internal carotid arteries (ICA) and their proximal branches associated with the compensatory development of a fragile collateral vessels network at the base of the brain (moyamoya vessels). MA 
is sub-classed in moyamoya disease (MMD) when MA is bilateral and it is the sole disease manifestation, or moyamoya syndrome (MMS) when MA is associated with a well-defined acquired or genetic condition. MA occurring unilaterally has been recently defined as unilateral moyamoya disease (UMMD) [1].

The pathogenesis of MA is unknown. Anomalies in angiogenesis and vasculogenesis, due to the detection of increased cytokine and growth factor concentrations have been invoked as potential disease mechanisms. Also genetic factors, given the high familial rate and the ethnic differences, are believed to be involved $[1,2]$.

The disease is rare with a prevalence being close to 3/100000 in East Asia and ten times less in Europe [3, 4]. MA mainly present intracranial hemorrhage or ischemic events in adults, whereas transient ischemic attacks (TIAs) or ischemic strokes occur in most symptomatic MA children [5]. Cerebral magnetic resonance imaging (MRI) can show ischemic and haemorrhagic lesions even in the absence of symptoms. The compensatory arterial moyamoya network and the intracranial and extracranial collaterals are best visualized on cerebral conventional angiography, which is still considered as the gold standard for MA diagnosis, according to established criteria $[6,7]$. Besides, non-invasive imaging, such as MRI-angiography (MRA) and computed tomography-angiography (CTA) allowing visualization of steno-occlusive lesions, are used for disease suspicion and follow-up monitoring [1]. Although no treatment of proven efficacy for reversing the primary stenotic disease process in MA is currently available, surgical revascularization has been shown to improve cerebral blood flow to the affected hemisphere(s) and prevent stroke [8-10]. A major issue is that the natural history of MA remains in most patients unpredictable. In addition, factors influencing the variable severity of the clinical course are still unknown due to the limited prospective literature data in non-operated cases $[1,11]$. These data are much more limited in western populations [12-15].

GENetics of mOyaMoyA (GEN-O-MA) project is a retrospective and prospective multicenter cohort study implemented in Italy aimed at creating a network of centers involved in MA care and research, and at collecting a large series of well characterized patients. This network integrates the experience of neurologists, neuroradiologists, neurosurgeons, biologists, and geneticists in MA study and care. An accurate and detailed clinical and neuroimaging classification will allow to describe the disease clinical features and disease course in Italy. Moreover, the collection of a large bio-repository of biological samples will allow the identification potential biological markers and genetic factors associated with the disease susceptibility. The present paper reports the most important methodological issues of the network and preliminary results.

\section{Patients and methods}

\section{Study design}

The study was designed as a multicenter (prospective and retrospective) observational cohort study across Italy. The project is coordinated by the Cerebrovascular Unit of the Fondazione IRCCS Istituto Neurologico Carlo Besta, Milan, Italy. A strong collaborative relation with the Neuroradiological, Neurosurgical, and Child Neuropsychiatric Unit and Developmental Neurology Division of the same hospital and with other participating centers allows a multidisciplinary collaboration leading to an improved result achievement.

Two independent advisory boards (Prof. Med. Peter Vajkoczy - Department of Neurosurgery, Charite Universitätsmedizin, Berlin, Germany, and Prof. Med. E. Tournier Lasserve - Department of Genetics, INSERM and University Paris Diderot, Paris, France) with an extensive expertise in the field of MA have been announced to monitor the study design and its reliability.

\section{Ethical issues}

The study design was approved by the Ethical Committee of the Fondazione IRCCS Istituto Neurologico Carlo Besta Milan, Italy (report no. 12, 10/01/2014), and of each participating center. The study was also performed in accordance with the World Medical Association Declaration of Helsinki.

Since it was designed as a pure observational study, patients underwent diagnostic procedures and received therapy according to local practice and disease guidelines. Informed written consent for study participation (for data and biological samples collection) from all patients or their legal representatives was mandatory for study inclusion.

\section{Study population}

The study population consists of a series of MA patients including MMD, MMS, and UMMD (aged between 0 and 75 years), collected both retrospectively and prospectively by the referring physicians of the centers participating in the study. MA was diagnosed according to established criteria: (1) stenosis or occlusion at the terminal portion of the ICA and/or the proximal portion of the anterior and/or middle cerebral arteries (MCA), (2) abnormal vascular networks in the vicinity of the stenotic lesions, and (3) a bilateral presence of these findings $[6,16]$.

A first sample of $100 \mathrm{MA}$ patients has been planned for clinical and neuroradiological assessment. Patients are being followed up for 2 years. 
Table 1 Clinical and neuroradiological data collected

Collected data of GEN-O-MA project

Demographic (age, age at onset, sex, ethnicity, region of origin)

Clinical symptoms of qualifying event (TIA, stroke, ICH)

Stroke severity and disability scales (NIHSS, mRS, Karnofsky Scale)

Disease clinical manifestations (migraine, seizures, cognitive impairment, psychiatric disorders, ophthalmic disease)

Vascular and lifestyle risk factors (hypertension, diabetes, smoke, atrial fibrillation, ischemic cardiopathy, alcohol intake, BMI $>25$, radiotherapy, traumatic brain injury)

Autoimmune disorders and history of associated conditions or syndromic features (i.e., hormone disorders, Down syndrome,

Neurofibromatosis, Marfan syndrome, sickle cell disease, mental retardation, dysmorphisms, artery dissections, aneurisms, coagulation disorders)

Familial history for MA, cerebrovascular events, and other neurological manifestations (migraine, cognitive impairments, psychiatric disorders, seizures, artery dissections, aneurisms)

Diagnostic workup (MRI, MRA, CTA, cerebral angiography, MRI perfusion, TCD)

Neuroimaging features (size and location of infarctions, degree of vessel stenosis-occlusion, regional relative CBV, MTT, and TTP, unilateral/bilateral disease, modified Suzuki scoring, external carotid arteries collaterals, leptomeningeal collaterals, dilatation of the AChorA, PcomA/ICA ratio, cortical and basal neovascularization, TCD mean flow in the middle cerebral arteries

Medical therapy (anti-aggregants, antiepileptic drugs, statins, other)

Surgical intervention (date, type, and side effects)

Follow-up (cerebrovascular events, neuroradiological assessment)

$B M I$, body mass index; $C T A$, computed tomography angiography; $I C H$, intracerebral hemorrhage; $M A$, moyamoya angiopathy; $M R A$, magnetic resonance angiography; MRI, magnetic resonance imaging; $m R S$, modified Ranking Scale; NIHSS, National Istitute of Health Stroke Scale/Score; $T I A$, transient ischemic attack; TCD, Transcranial Doppler ultrasound, $C B V$, cerebral blood flow; $M T T$, mean transit time; TTP, time to peak; $A C h o r A$, Anteriori choroidal artery, $P$ comA, posterior communicating artery, ICA, internal carotid artery

\section{Clinical data and biological samples collection}

All included patients underwent a comprehensive workup including routine and autoimmunity blood tests, neuro-ophtalmologic and electrophysiological evaluation, Doppler/transcranial ultrasound, and neuroimaging examinations. A specific form implemented from current literature has been developed for standardized data collection. For each patient demographic and clinical data, cerebrovascular risk factors, familial antecedents, racial descents, current pathologies including immune diseases, genetic syndromes, and pharmacological treatment have been registered. Cerebrovascular events are classified according to established standardized criteria (i.e., hemorrhagic, ischemic, lesion site) based on clinical features and MRI findings. Also, other neurological clinical features associated with MA including cognitive deficits, seizures, and psychiatric disorders have been reported. The use of these standardized forms, the centralized data managing, and the control of quality assures a harmonized and homogeneous data collection across the participating centers. Clinical and neuroradiological data collected within the GEN-O-MA project are summarized in Table 1.

Blood and plasma samples from a subgroup of MA patients and age-sex matched healthy and unrelated (atherosclerotic patients) controls (HC and UC respectively) are being collected. Cerebrospinal fluid CSF samples and arterial specimens (MCA) of MA patients and UC are being also gathered in a subset of patients undergoing revascularization surgery.

\section{Central neuroimaging analysis}

The study provides for a centralized analysis of brain imaging studies, including contrast-enhanced magnetic resonance imaging (MRI), MR angiography, perfusion weighted imaging (PWI), digital subtraction angiography (DSA), and transcranial doppler (TCD) ultrasound. For patients who underwent brain MRI, sagittal T1-weighted spin echo, axial T2-weighted turbo spin echo, coronal T2-weighted FLAIR, diffusion weighted images (DWI), threedimensional time of flight phase contrast angiography, and three-dimensional T1-weighted gradient echo sequences after gadolinium injection were recommended. Two neuroradiologists (G.F and M.G.), with more than 7 years experience in neuroimaging visual rating and blinded to clinical and biological/genetic data are carried out quantitative and qualitative analyses, with reference to the shape, size and location of infarctions, and the degree of vessels stenosis-occlusion in the corresponding areas. Perfusion weighted images (PWI) were acquired on a $1.5 \mathrm{~T}$ or $3 \mathrm{~T}$ units, to evaluate the regional relative cerebral blood volume (CBV), relative mean transit time (MTT), and time to peak (TTP) maps. A comprehensive digital subtraction cerebral angiography (DSA) with selective 
catheterization of both internal and external carotid arteries and the dominant vertebral artery was mandatory to evaluate collateral circulation for MA diagnosis for study inclusion. MA was classified into the bilateral or unilateral type according to the number of the involved distal internal carotid arteries, observed on DSA. Disease severity was evaluated on DSA by applying a modified Suzuki scoring [16] on cerebral angiography findings, as follows: (0) no evidence of disease, (1) mild-to-moderate stenosis around internal carotid artery (ICA) bifurcation with absent or slightly developed ICA MMD, (2) severe stenosis around the ICA bifurcation or occlusion of either proximal anterior or MCA branches with well-developed ICA MMD, (3) occlusion of both anterior and MCA branches with well-developed ICA MMD (only a few of anterior or MCA branches or both are faintly opacified in antegrade fashion through meshwork of ICA MMD), and (4) complete occlusion of both anterior and MCA branches with absent or small amount of ICA MMD (without opacification of either anterior or MCA branches in antegrade fashion). [17] The external carotid artery collaterals were graded by using a 4-point scale: (0) no collateral distribution; (1) slight collateral distribution, often with dilution; (2) small but definite collateral supply; and (3) full collateral filling [18, 19].

In addition, we investigated the presence of leptomeningeal collaterals (LMC) coursing from the posterior cerebral artery (PCA) to the frontal, temporal or parietal lobes, the dilatation of the anterior choroidal artery (AChorA), and the posterior communicating artery (PcomA)/ICA ratio*.Cortical neovascularization was defined as an enlarged winding of the distal cortical arteries observed in the arterial DSA phase and categorized into anterior or posterior cortical neovascularization depending on their sources (anterior vs. posterior circulation). The degree of basal collaterals (BCs) was measured as the ratio of the height from the carotid T-junction to the end of the $\mathrm{BC}$ vessels and the height from the carotid T-junction to the highest point of the imaginary superior sagittal sinus in the antero-posterior projection $\times 100$.

Transcranial Doppler (TCD) was performed using a 2-MHz transducer to acquire a continuous measurement of mean flow velocities of the middle (M1-M2 segments whenever available), anterior (A1 segment, whenever available) and posterior (P1-P2 segments) cerebral arteries, basilar artery, and intracranial vertebral arteries. Moreover, an evaluation of vasomotor reactivity through breath-holding technique from the middle cerebral artery territory was bilaterally obtained in case of adequate bone temporal windows [20, 21].

\section{Experimental and genetic analysis}

Blood, DNA, and CSF samples are being collected, when available, to evaluate (1) number and function of circulating endothelial progenitor cells (cEPCs), (2) levels of growth
Fig. 1 Clinical center participating to the GEN-O-MA project: (1) Fondazione IRCCS Istituto Neurologico Carlo Besta, Milano; (2) ASST- Spedali Civili Di Brescia, Brescia; (3) ASST Papa Giovanni XXIII, Bergamo; (4) Fondazione IRCCS Ca' Granda - Ospedale Maggiore Policlinico, Milano; (5) ASST Grande Ospedale Metropolitano Niguarda, Milano; (6) Ospedale ASST Settelaghi Varese, Varese; (7) ASST Ovest Milanese, Ospedale di Legnano; (8) Ospedale Santa Maria Della Misericordia di Udine, Udine; (9) Arcispedale Santa Maria Nuova, Reggio Emilia; (10) Nocsae Modena, Modena; (11) Azienda Ospedaliera Universita’ Degli Studi di Padova, Padova; (12) Clinica Neurologica Padova, Padova; (13) IRCCS Istituto Giannina Gaslini, Genova; (14) Azienda Ospedaliera Universitaria Careggi, Firenze; (15) Umberto I- Policlinico di Roma, Roma; (16) Policlinico Universitario Campus Bio-Medico, Roma; (17) Ospedale Santa Maria Della Misericordia, Perugia; (18) Ospedale Regionale San Salvatore, L'aquila; (19) Ospedale di San Benedeto Del Tronto, San Benedetto Del Tronto.

factor within plasma and CSF, (3) MCA gene expression profile of angiogenic and growth factors coding genes, and (4) genetic factors associated with sporadic and familial MA cases.

Levels of cEPCs are being evaluated in whole blood samples by flow cytometry [22] and by flow-count fluorospheres (Beckman Coulter s.r.1., Cassina De' Pecchi, Italy). CD45dimCD34 + CD133 + mononuclear cells will be considered as cEPCs. Moreover, in order to normalize the absolute number of cEPCs on the number of circulating leukocytes, a complete white blood cell (WBC) count is performed applying the Advia 120 cell counter (Bayer, Leverkusen, Germany). The \% of cEPCs will be calculated as follows: $\%$ $\mathrm{cEPCs}=$ no. $\mathrm{cEPC} \times 100 /($ no. $\mathrm{WBC} / \mu \mathrm{l})$. The EPC function will be assessed in vitro by isolating peripheral blood mononuclear cells (PB-MNCs) as previously reported [23]. The cell number for $\mathrm{mm}^{2}$ in vitro (cells $/ \mathrm{mm}^{2}$ ) are being evaluated at day $3,7,10,17$, and 31. Conditioned medium (CM) will be used as stimulus for tube formation assays at day 7 , and the angiogenic EPC activity will be tested at day 17 . Finally, realtime (RT)-PCR experiments is being performed to evaluate the gene expression of endothelial markers and angiogenic growth factors in PB-MNCs and in EPC in vitro cultures (HC versus MA).

MA plasma and available CSF samples will be tested for cytokine and growth factor levels by using enzyme-linked immunosorbent assays (ELISA; R\&D Systems, Minnesota, USA) and Bio-Plex Assays (Bio-Rad Laboratories, Segrate, Italy).

Total RNA will be extracted from affected MCA and the respective UC arteries followed by RT-PCR experiments using the 96-Well RT ${ }^{2}$ Profiler ${ }^{\mathrm{TM}}$ Human Angiogenic Growth Factors PCR Array (PAHS-072ZD) (Qiagen, Hilden, Germany).

For genetic analysis, genomic DNA are being obtained from peripheral blood of patients and HCs using QIAamp DNA Mini Kit (Qiagen, Hilden, Germany). A two-step analysis has been planned including the genetic screening of RNF213 gene of all patients, whereas exome sequencing analysis was intended for familial cases. 
ASST Spedali Civili Di Brescia, Brescia

ASST Papa Giovanni XXIII, Bergamo

ASST Ovest Milanese, Legnano

Fondazione IRCCS Istituto Neurologico Carlo Besta, Milano

ASST Grande Ospedale Metropolitano Niguarda, Milano

Fondazione IRCCS Ca' Granda - Opsedale Maggiore Policlinico, Milano

Ospedale ASST Settelaghi Varese, Varese

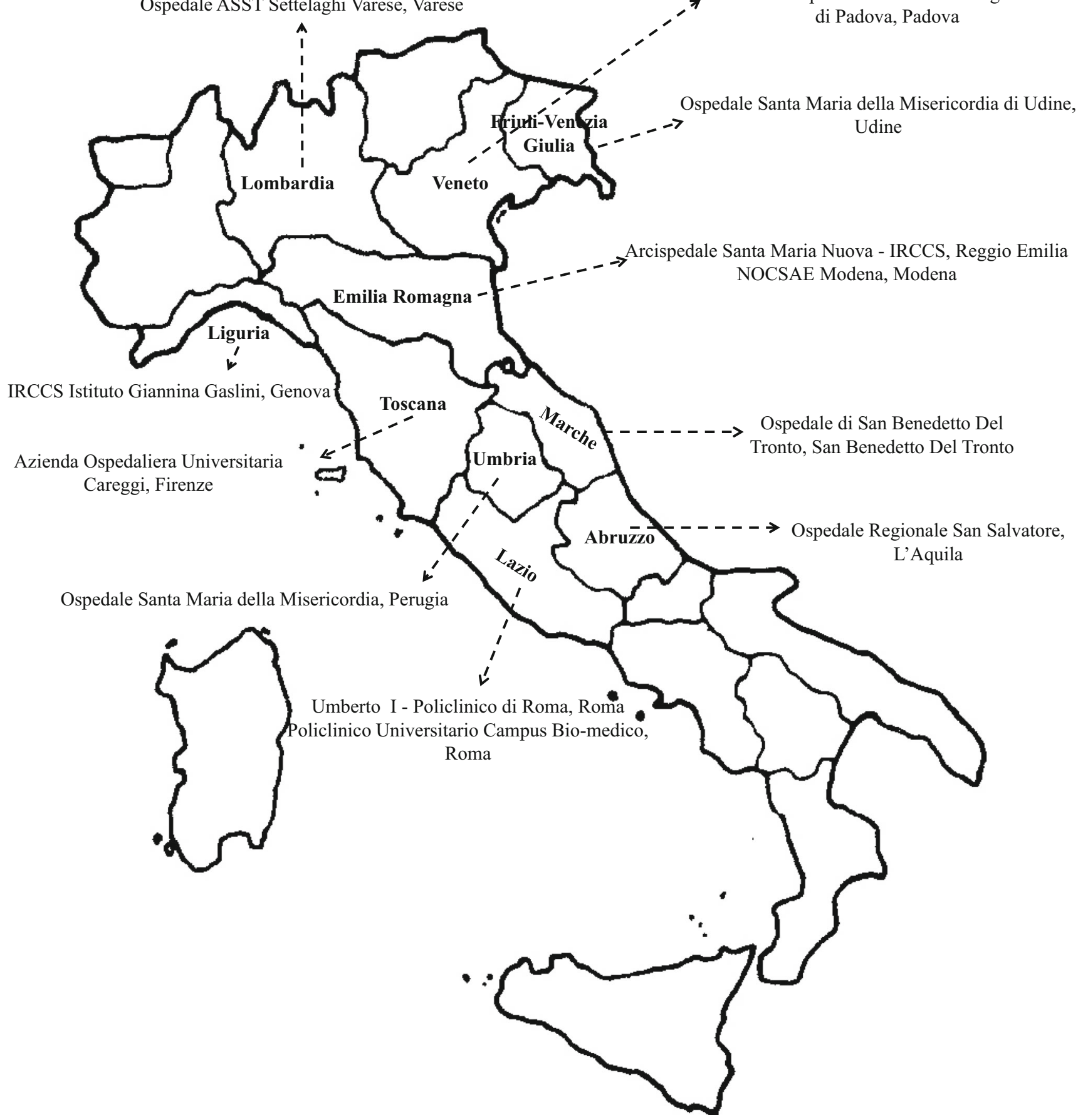

\section{Statistical data analysis}

Before starting the analysis, a quality evaluation of all collected data will be performed and the validity of screening
Clinica Neurologica Padova, Padova Azienda Ospedaliera Università degli Studi di Padova, Padova 
uMMD, or MA grading) with respect to the clinical and neurological features as well as to the results of biological experiments and genetic analysis. The independence of single predictive factors will be assessed by logistic regression analysis. All analyses will be calculated using STATA 8.0 (StataCorp LP, College Station, TX) and S-PLUS (Suite 44, Level 9, 88 Pitt Street Sydney New South Wales, Australia).

\section{Results}

\section{Study centers}

Nineteen centers effectively participated in MA patients recruitment (Fig. 1). Although the recruiting period was intended to start in January 2015, patient data collection began almost 6 months later than the anticipated date due to awaiting the local ethics committee approval.

\section{Patients' characteristics}

Between January 2015 and July 2017, 98 MA patients (MMD, MMS) mean \pm SD age $35.5 \pm 19.6$ years were collected. Seventy-three (74.5\%) were adults and 25 (25.5\%) were children. Mean age \pm SD of the onset was $33.5 \pm 19.8$ years and $67(68.4 \%)$ were females. Mean NIHSS \pm SD and $\mathrm{mRS}$ $\pm \mathrm{SD}$ were $2.95 \pm 3.24$ and $0.70 \pm 1$, respectively.

Twenty-six cases $(26.5 \%)$ presented high blood pressure, $25(25.5 \%)$ hypercholesterolemia, $7(7.1 \%)$ had diabetes, while as expected, atrial fibrillation and cardiopathy were present in $3.1 \%$ of our MA cases.

The index event was stroke in $64(65.3 \%)$ cases $(76.5 \%$ ischemic and $23.4 \%$ hemorrhagic) and TIA in (12.2\%) cases. The number of cases for center is represented in Fig. 2. Sixtyfour $(65.3 \%)$ patients exhibited only one cerebrovascular event at the inclusion time. Only 20 patients referred $\geq 2$ cerebrovascular event in the past.

Forty-five patients $(45.9 \%)$ presented headache/migraine, 29 (29.6\%) seizures, 21 (21.4\%) cognitive impairments, 20 (20.4\%) psychiatric disorders, and $10(10.2 \%)$ ophthalmic disorders.

Bilateral involvement was identified in 60 (61.2\%) patients, and $41(41.8 \%)$ patients underwent surgical revascularization.

Familial history for MA was present in 4 (4.1\%) of the patients whereas familial history for stroke/TIA was present in $33(33.7 \%)$ of the cases. Preliminary GEN-O-MA clinical data and reported literature from Caucasian and Asian MA patients are outlined in Table 2.

\section{Moyamoya biorepository}

For cEPC analysis whole blood of $16 \mathrm{MA}, 20 \mathrm{HC}$, and $8 \mathrm{UC}$ was collected till date, whereas $8 \mathrm{MA}$ versus $5 \mathrm{UC}$ affected arteries were analyzed for $\mathrm{RT}^{2}$ Profiler ${ }^{\mathrm{TM}}$ Human Angiogenic Growth Factors PCR Array.

To date, 27 plasma and 15 CSF samples along with $30 \mathrm{MA}, 20 \mathrm{HC}$, and $8 \mathrm{UC}$ DNA samples have been collected.

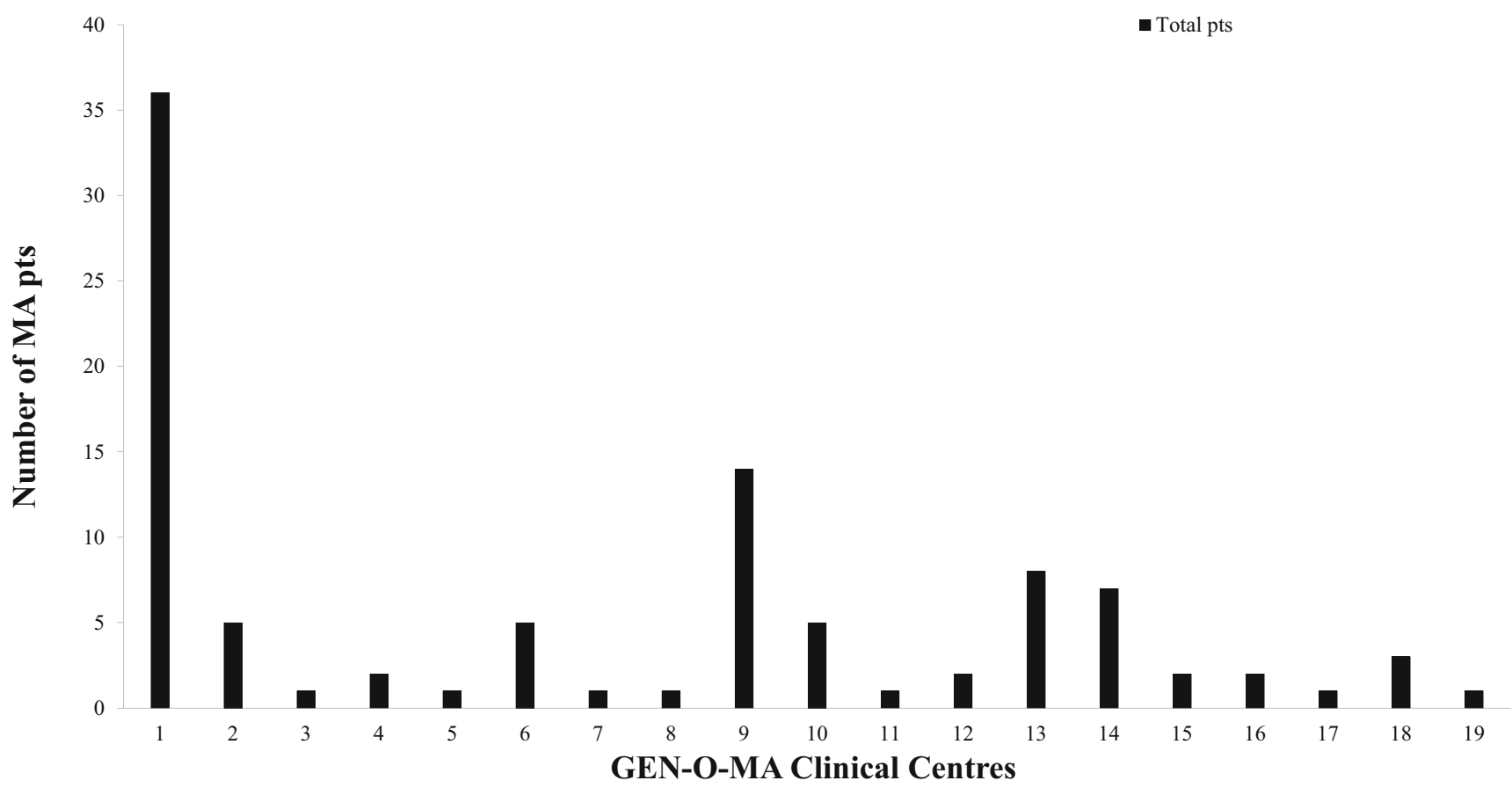

Fig. 2 Number of patients for center participating to the GEN-O-MA project 
Table 2 Preliminary clinical data of GEN-O-MA vs. European and Asian MA patients

$$
\text { Caucasian patients Asian patients }
$$

Demographic characteristics

\begin{tabular}{|c|c|c|c|c|c|c|c|c|}
\hline \multirow[t]{2}{*}{ Clinical features } & \multirow{2}{*}{$\begin{array}{l}\text { GEN-O-MA } \\
(n=98)\end{array}$} & \multicolumn{2}{|c|}{ European $[12,15]$} & \multicolumn{2}{|c|}{ American $[24,25]$} & \multirow{2}{*}{$\begin{array}{l}\text { Chinese [5] } \\
(n=470)\end{array}$} & \multirow{2}{*}{$\begin{array}{l}\text { Japanese [26] } \\
(n=941)\end{array}$} & \multirow{2}{*}{$\begin{array}{l}\text { Korean [27] } \\
(n=175)\end{array}$} \\
\hline & & $(n=153)$ & $(n=61)$ & $(n=7473)$ & $(n=94)$ & & & \\
\hline $\begin{array}{l}\text { Age at onset } \\
(\text { mean } \pm \mathrm{SD} \text {; year })\end{array}$ & $33.5 \pm 19.8$ & $35.8 \pm 14.86$ & $31.5 \pm 17.9$ & - & 34.5 & 36.8 & - & $42.2 \pm 11.3$ \\
\hline Female:male & $2.2: 1$ & $2.9: 1$ & $4.5: 1$ & $2.2: 1$ & $2.6: 1$ & $1: 1$ & $1.98: 1$ & $1.57: 1$ \\
\hline Adults & $74.5 \%$ & $83.7 \%$ & $83.6 \%$ & $70.1 \%$ & $100 \%$ & $100 \%$ & - & $100 \%$ \\
\hline Children & $25.5 \%$ & $16.3 \%$ & $16.4 \%$ & $29.9 \%$ & $0 \%$ & $0 \%$ & - & $0 \%$ \\
\hline \multicolumn{9}{|c|}{ Index event characteristics } \\
\hline Stroke & $65.3 \%$ & - & $63.9 \%$ & $18.1 \%$ & $73.4 \%$ & $26.8 \%$ & $41 \%$ & $94.3 \%$ \\
\hline Ischemic & $50 \%$ & $81 \%$ & $50.8 \%$ & $11.7 \%$ & $57.4 \%$ & - & $20 \%$ & $45.1 \%$ \\
\hline Hemorrhagic & $15.3 \%$ & $8.5 \%$ & $13.1 \%$ & $6.4 \%$ & $16.0 \%$ & $20.4 \%$ & $21 \%$ & $54.9 \%$ \\
\hline TIA & $11.2 \%$ & - & $6.6 \%$ & $34.0 \%$ & $34 \%$ & $41.1 \%$ & $46 \%$ & - \\
\hline \multicolumn{9}{|l|}{ Other symptoms/signs } \\
\hline Headache & $45.9 \%$ & $3.9 \%$ & $11.5 \%$ & - & $21.3 \%$ & $9.6 \%$ & $6 \%$ & $29.7 \%$ \\
\hline Seizures & $29.6 \%$ & $1.3 \%$ & $4.9 \%$ & - & - & $0.9 \%$ & $4 \%$ & $2.9 \%$ \\
\hline Psychiatric disorders & $20.1 \%$ & - & - & - & - & - & - & - \\
\hline Cognitive impairment & $22.4 \%$ & $1.3 \%$ & - & - & $5.3 \%$ & - & - & $5.1 \%$ \\
\hline Ophthalmic diseases & $36.4 \%$ & - & - & - & - & - & - & $9.1 \%$ \\
\hline Autoimmune diseases & $16.3 \%$ & - & - & - & $22.3 \%$ & - & - & - \\
\hline \multirow{2}{*}{\multicolumn{9}{|c|}{$\begin{array}{l}\text { Anatomic extent of moyamoya } \\
\text { side }\end{array}$}} \\
\hline & & & & & & & & \\
\hline Bilateral & $61.2 \%$ & $77.8 \%$ & - & - & $86.2 \%$ & $88.9 \%$ & - & - \\
\hline
\end{tabular}

BMI, body mass index; MA, moyamoya angiopathy; NIHSS, National Institute of Health Stroke Scale/Score; TIA, transient ischemic attack

\section{Discussion}

The exact phenotype and clinical history of MA are still poorly defined. The remarkable disease variability and the small size reported in studies addressing the disease pathogenesis along with inadequate data on brain imaging follow-up limit a clear definition of the disease course and the identification of factors influencing MA progression [28]. Particularly, data on MA in Western countries, hereby mostly in Europe are lacking. Although a similar phenotype between the US and European patients has been described, the rarity of the disease in Europe, the lack of systematic studies, the multi-ethnic origin, and the heterogeneity of the studied cohorts further impair the acquisition of clear information on clinical feature and disease progression in Europeans [12-15, 28-32].

The identification of clinical and imaging predictors is necessary to improve a prognostic evaluation and develop innovative therapeutic approaches. Moreover, pathophysiological mechanisms driving MA are largely unknown. Based on the existence of familial cases and the observation of a pronounced ethnicity effect, a genetic contribution is likely presumed $[1,11,14,28]$. Strong association between MA and a RNF213 variant (R4810K) has been found in the East Asian population in around $90 \%$ of familial and $70 \%$ of sporadic cases. Despite preliminary reports excluded an association between this gene and MA in Caucasians [30]. Recently, rare RNF213 variants have been reported also in some European cases, particularly with early onset and familial MA [31]. Additional familial cases of MA associated with facial dimorphisms and achalasia respectively were recently associated mutations in BRCC3 deubiquitinase and GUCY1A3 gene, encoding the major nitric oxide receptor in vascular smooth muscle cells [32, 33]. However, these genes are not able to completely explain MA pathogenesis, and the genetic background of MA remains still not identified.

Several studies reported an overexpression of proangiogenic factors in the CSF, blood, or tissue samples of MA patients supporting the hypothesis that an unbalance of these factors may contribute to an impaired angiogenic and vasculogenic mechanisms leading to the characteristic vessel fragility [2, 34].

Poor understanding of the disease determinants limited so far advances in the clinical care and the development of innovative and personalized treatment. Until date, no 
treatment has been identified to impair or slow the development of stenotic process and fragile collaterals. The direct or indirect revascularization surgery is considered as the best therapeutic option to reduce the occurrence of cerebrovascular events [11]. Although a recent metaanalysis has confirmed the surgical revascularization to prevent recurrent strokes in adult MMD patients, the risks, benefits, and indications for surgery are still uncertain and influenced by the retrospective design of most included studies [10]. Moreover, the differences in the selection of patient cohorts, diagnostic steps, surgical techniques, related to the clinical experience of single center limit the relevance of the available data. In summary, controlled trials prospectively analyzing the efficacy of different treatment strategies, by applying standardized clinical assessments, are largely needed [11].

The GEN-O-MA project is, to our knowledge, the first Italian Network and one of the largest Europeans studies [12-15] published so far. The objective of this network, which is integrated with other large European clinical centers involved in MA study, is to describe the clinical phenotype and identify disease progression markers of MA by applying a careful clinical and neuroradiological evaluation. Secondarily it is aimed at understanding the pathophysiology of MA by applying a translational biological approach.

At a preliminary analysis of our MA series, although the comparison with previous European and American series is difficult, due to the lack and incompleteness of previously collected data, we confirmed a similar age of onset and the higher female predominance, with a female/male ratio more comparable to the German and American $[12,24,25]$ rather than to the Finnish population. The occurrence of ischemic and hemorrhagic stroke and clinical features frequency was alike, except for a modest increase rate of headache and seizures $[12,15$, 25] (Table 2).

Our study has some important strength points: (1) it is a multicenter study with a systematic and standardized recruitment, (2) it permits a careful phenotyping by applying a detailed questionnaire and a standardized qualitative and quantitative characterization based on imaging data, and (3) it is characterized by an innovative experimental approach, including the evaluation of biological and genetic markers by analyzing affected tissue samples. The correlation between clinical and biological data as well as the direct analysis of affected vessel specimens makes our approach highly promising [34].

The principal limitation of the study may be the insufficient sample size, due to the high analysis costs, which may impair the understanding the significance of disease progression markers and the role of rare variants with very small effect sizes and weak gene-environment interactions.
However, a collaborative effort allowing to collect a large clinical dataset as well as DNA of MA patients, the GEN-OMA project is expected to provide novel data on genetic susceptibility of MA within the Italian and/or European population. Due to the large cohort of Caucasian patients, stratified by specific subclasses, our study allows the identification of individuals with a potential risk for the development of MA. [23]. The identification of phenotypes at-risk and/or specific biological players driving the disease may improve our ability to specify the disease prognosis for individual patients and to develop personalized intervention/treatment options.

\section{Compliance with ethical standards}

Conflict of interest The authors declare that they have no conflict of interest.

Informed consent Informed consent was obtained from all individual participants included in the study.

Publisher's note Springer Nature remains neutral with regard to jurisdictional claims in published maps and institutional affiliations.

\section{References}

1. Bersano A, Guey S, Bedini G, Nava S, Hervé D, Vajkoczy P, Tatlisumak T, Sareela M, van der Zwan A, Klijn CJ, Braun KP, Kronenburg A, Acerbi F, Brown MM, Calviere L, Cordonnier C, Henon H, Thines L, Khan N, Czabanka M, Kraemer M, Simister R, Prontera P, Tournier-Lasserve E, Parati E, European Moyamoya Initiative (2016) Research progresses in understanding the pathophysiology of moyamoya disease. Cerebrovasc Dis 41:105-118

2. Bedini G, Blecharz KG, Nava S, Vajkoczy P, Alessandri G, Ranieri M, Acerbi F, Ferroli P, Riva D, Esposito S, Pantaleoni C, Nardocci N, Zibordi F, Ciceri E, Parati EA, Bersano A (2016) Vasculogenic and angiogenic pathways in moyamoya disease. Curr Med Chem 23:315-345

3. Kleinloog R, Regli L, Rinkel GJ, Klijn CJ (2012) Regional differences in incidence and patient characteristics of moyamoya disease: a systematic review. J Neurol Neurosurg Psychiatry 83:531-536

4. Yonekawa Y, Ogata N, Kaku Y, Taub E, Imhof HG (1997) Moyamoya disease in Europe, past and present status. Clin Neurol Neurosurg 99(Suppl 2):S58-S60

5. Bao XY, Duan L, Li DS, Yang WZ, Sun WJ, Zhang ZS, Zong R, Han C (2012) Clinical features, surgical treatment and long-term outcome in adult patients with Moyamoya disease in China. Cerebrovasc Dis 34:305-313

6. Fukui M (1997) Guidelines for the diagnosis and treatment of spontaneous occlusion of the circle of Willis ('moyamoya' disease). Research Committee on Spontaneous Occlusion of the Circle of Willis (Moyamoya Disease) of the Ministry of Health and Welfare, Japan. Clin Neurol Neurosurg 99(Suppl 2):S238-S240

7. Research Committee on the Pathology and Treatment of Spontaneous Occlusion of the Circle of Willis; Health Labour Sciences Research Grant for Research on Measures for Infractable Diseases (2012) Guidelines for diagnosis and treatment of moyamoya disease (spontaneous occlusion of the circle of Willis). Neurol Med Chir (Tokyo) 52:245-266 
8. Ishikawa T, Houkin K, Kamiyama H, Abe H (1997) Effects of surgical revascularization on outcome of patients with pediatric moyamoya disease. Stroke 28:1170-1173

9. Starke RM, Komotar RJ, Connolly ES (2009) Optimal surgical treatment for moyamoya disease in adults: direct versus indirect bypass. Neurosurg Focus 26:E8

10. Jeon JP, Kim JE, Cho WS, Bang JS, Son YJ, Oh CW (2017) Metaanalysis of the surgical outcomes of symptomatic moyamoya disease in adults. J Neurosurg 5:1-7

11. Guey S, Tournier-Lasserve E, Hervé D, Kossorotoff M (2015) Moyamoya disease and syndromes: from genetics to clinical management. Appl Clin Genet 8:49-68

12. Acker G, Goerdes S, Schneider UC, Schmiedek P, Czabanka M, Vajkoczy P (2015) Distinct clinical and radiographic characteristics of moyamoya disease amongst European Caucasians. Eur J Neurol 22:1012-1017

13. Kraemer M, Heienbrok W, Berlit P (2008) Moyamoya disease in Europeans. Stroke 39:3193-3200

14. Krischek B, Kasuya H, Khan N, Tatagiba M, Roder C, Kraemer M (2011) Genetic and clinical characteristics of Moyamoya disease in Europeans. Acta Neurochir Suppl 112:31-34

15. Saarela M, Mustanoja S, Pekkola J, Tyni T, Hernesniemi J, Kivipelto L, Tatlisumak T (2017) Moyamoya vasculopathy - patient demographics and characteristics in the Finnish population. Int J Stroke 12:90-95

16. Suzuki J, Takaku A (1969) Cerebrovascular 'moyamoya' disease. Disease showing abnormal net-like vessels in base of brain. Arch Neurol 20:288-299

17. Czabanka M, Peña-Tapia P, Schubert GA, Heppner FL, Martus P, Horn P, Schmiedek P, Vajkoczy P (2011) Proposal for a new grading of Moyamoya disease in adult patients. Cerebrovasc Dis 32:41-50

18. Chung JW, Kim SJ, Bang OY, Kim KH, Ki CS, Jeon P, Yeon JY, Kim JS, Hong SC, Shin HJ (2016) Determinants of basal collaterals in moyamoya disease: clinical and genetic factors. Eur Neurol 75: 178-185

19. Strother MK, Anderson MD, Singer RJ, Du L, Moore RD, Shyr Y, Ladner TR, Arteaga D, Day MA, Clemmons PF, Donahue MJ (2014) Cerebrovascular collaterals correlate with disease severity in adult North American patients with Moyamoya disease. AJNR Am J Neuroradiol 35:1318-1324

20. Silvestrini M, Troisi E, Matteis M, Cupini LM, Bernardi G (1996) Effect of smoking on cerebrovascular reactivity. J Cereb Blood Flow Metab 16:746-749

21. Markus HS, Harrison MJ (1992) Estimation of cerebrovascular reactivity using transcranial Doppler, including the use of breathholding as the vasodilatatory stimulus. Stroke 23:668-673

22. Corsini E, Ciusani E, Gaviani P, Silvani A, Canazza A, Bernardi G, Calatozzolo C, DiMeco F, Meco FD, Salmaggi A (2012) Decrease in circulating endothelial progenitor cells in treated glioma patients. J Neuro-Oncol 108:123-129
23. Asahara T, Murohara T, Sullivan A, Silver M, van der Zee R, Li T, Witzenbichler B, Schatteman G, Isner JM (1997) Isolation of putative progenitor endothelial cells for angiogenesis. Science 275: 964-967

24. Kainth D, Chaudhry SA, Kainth H, Suri FK, Qureshi AI (2013) Epidemiological and clinical features of moyamoya disease in the USA. Neuroepidemiology 40(4):282-287

25. Bower RS, Mallory GW, Nwojo M, Kudva YC, Flemming KD, Meyer FB (2013) Moyamoya disease in a primarily white, midwestern US population: increased prevalence of autoimmune disease. Stroke 44(7):1997-1999

26. Hoshino H, Izawa Y, Suzuki N (2012) Research committee on Moyamoya disease. Epidemiological features of moyamoya disease in Japan. Neurol Med Chir (Tokyo) 52(5):295-298

27. Jang DK, Lee KS, Rha HK, Huh PW, Yang JH, Park IS, Ahn JG, Sung JH, Han YM (2014) Clinical and angiographic features and stroke types in adult moyamoya disease. AJNR Am J Neuroradiol 35(6):1124-1131

28. Kuroda S, Houkin K (2008) Moyamoya disease: current concepts and future perspectives. Lancet Neurol 7:1056-1066

29. Ganesan V, Smith ER (2015) Moyamoya: defining current knowledge gaps. Dev Med Child Neurol 57:786-787

30. Liu W, Senevirathna ST, Hitomi T, Kobayashi H, Roder C, Herzig R, Kraemer M, Voormolen MH, Cahová P, Krischek B, Koizumi A (2013) Genomewide association study identifies no major founder variant in Caucasian moyamoya disease. J Genet 92:605-609

31. Guey S, Kraemer M, Hervé D, Ludwig T, Kossorotoff M, Bergametti F, Schwitalla JC, Choi S, Broseus L, Callebaut I, Genin E, Tournier-Lasserve E, FREX consortium (2017) Rare RNF213 variants in the C-terminal region encompassing the RING-finger domain are associated with moyamoya angiopathy in Caucasians. Eur J Hum Genet 25:995-1003

32. Miskinyte $\mathrm{S}$, Butler MG, Hervé D, Sarret C, Nicolino M, Petralia JD, Bergametti F, Arnould M, Pham VN, Gore AV, Spengos K, Gazal S, Woimant F, Steinberg GK, Weinstein BM, TournierLasserve E (2011) Loss of BRCC3 deubiquitinating enzyme leads to abnormal angiogenesis and is associated with syndromic moyamoya. Am J Hum Genet 88:718-728

33. Hervé D, Philippi A, Belbouab R, Zerah M, Chabrier S, CollardeauFrachon S, Bergametti F, Essongue A, Berrou E, Krivosic V, SainteRose C, Houdart E, Adam F, Billiemaz K, Lebret M, Roman S, Passemard S, Boulday G, Delaforge A, Guey S, Dray X, Chabriat H, Brouckaert P, Bryckaert M, Tournier-Lasserve E (2014) Loss of $\alpha 1 \beta 1$ soluble guanylate cyclase, the major nitric oxide receptor, leads to moyamoya and achalasia. Am J Hum Genet 94:385-394

34. Blecharz KG, Frey D, Schenkel T, Prinz V, Bedini G, Krug SM, Czabanka M, Wagner J, Fromm M, Bersano A, Vajkoczy P (2017) Autocrine release of angiopoietin-2 mediates cerebrovascular disintegration in Moyamoya disease. J Cereb Blood Flow Metab 37: $1527-1539$

\section{Affiliations}

Anna Bersano ${ }^{1}$ (D) $\cdot$ Gloria Bedini $^{2} \cdot$ Sara Nava ${ }^{2} \cdot$ Francesco Acerbi $^{3} \cdot$ Davide Rossi Sebastiano $^{4} \cdot$ Simona Binelli $^{4}$. Silvana Franceschetti ${ }^{4}$. Giuseppe Faragò ${ }^{5}$ - Marina Grisoli ${ }^{6}$. Andrea Gioppo ${ }^{5}$. Paolo Ferroli ${ }^{3}$. Maria Grazia Bruzzone ${ }^{6} \cdot$ Daria Riva $^{7}$ - Elisa Ciceri ${ }^{6}$. Chiara Pantaleoni ${ }^{7} \cdot$ Veronica Saletti $^{7} \cdot$ Silvia Esposito $^{7}$. Nardo Nardocci ${ }^{8} \cdot$ Federica Zibordi $^{8} \cdot$ Luigi Caputi $^{1}$ - Stefania Bianchi Marzoli ${ }^{9}$. Maria Luisa Zedde ${ }^{10}$. Marco Pavanello ${ }^{11}$ - Alessandro Raso ${ }^{11}$ • Valeria Capra ${ }^{11}$ - Leonardo Pantoni ${ }^{12}$. Cristina Sarti $^{13}$ - Alessandro Pezzini ${ }^{14}$. Filomena Caria ${ }^{14}$. Maria Luisa Dell' Acqua ${ }^{15}$. Andrea Zini ${ }^{15}$. Claudio Baracchini ${ }^{16}$. Filippo Farina $^{16}$.

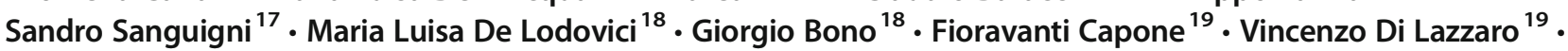
Silvia Lanfranconi ${ }^{20}$. Massimiliano Toscano ${ }^{21}$ - Vittorio Di Piero ${ }^{21}$. Simona Sacco ${ }^{22}$. Antonio Carolei ${ }^{22}$. 
Danilo Toni $^{21}$ • Maurizio Paciaroni ${ }^{23} \cdot$ Valeria Caso $^{23} \cdot$ Patrizia Perrone $^{24} \cdot$ Maria Vittoria Calloni $^{24} \cdot$ Alfredo Romani $^{25}$. $^{2}$ Marco Cenzato ${ }^{26}$. Alessia Fratianni ${ }^{26}$. Emilio Ciusani ${ }^{27}$ • Paolo Prontera ${ }^{28}$ • Elisabeth Tournier Lasserve ${ }^{29}$. Kinga Blecharz ${ }^{30}$. Peter Vajkoczy ${ }^{30}$. Eugenio Agostino Parati ${ }^{1}$

1 Cerebrovascular Unit, Neurological Institute "C. Besta" IRCCS Foundation, Milan, Italy

2 Laboratory of Cellular Neurobiology, Fondazione IRCCS Istituto Neurologico Carlo Besta, Milan, Italy

3 Neurosurgical Unit, Fondazione IRCCS Istituto Neurologico Carlo Besta, Milan, Italy

4 Neurophysiopathology Department and Epilepsy Centre, Fondazione IRCCS Istituto Neurologico Carlo Besta, Milan, Italy

5 Diagnostic Imaging Department \& Interventional Neuroradiology, Fondazione IRCCS Istituto Neurologico Carlo Besta, Milan, Italy

6 Neuroradiological Unit, Fondazione IRCCS Istituto Neurologico Carlo Besta, Milan, Italy

7 Developmental Neurology Division, Fondazione IRCCS Istituto Neurologico Carlo Besta, Milan, Italy

8 Department of Child Neurology, Fondazione IRCCS Istituto Neurologico Carlo Besta, Milan, Italy

9 Neuropthalmologic Unit, IRCCS Istituto Auxologico, Milan, Italy

10 Neurology Unit, Stroke Unit, Azienda Unità Sanitaria LocaleIRCCS di Reggio Emilia, Reggio Emilia, Italy

11 Neurosurgery Unit, Istituto Giannina Gaslini, Genoa, Italy

12 L.Sacco Department of Biomedical and Clinical Science, University of Milan, Milan, Italy

13 NEUROFARBA Department Neuroscience Section, University of Florence, Florence, Italy

14 Department of Clinical and Experimental Sciences, Neurology Clinic, University of Brescia, Brescia, Italy

15 Stroke Unit, Nuovo Ospedale Civile S Agostino Estense, University Hospital of Modena, Modena, Italy

16 Stroke Unit and Neurosonology Laboratory, Department of Neurological Sciences, University of Padua School of Medicine, Padua, Italy
17 Department of Neurology, General Hospital Madonna del Soccorso, San Benedetto del Tronto, Italy

18 Stroke Unit Circolo Hospital and Macchi Foundation, Varese Hospital, Varese, Italy

19 Unit of Neurology, Neurophysiology, Neurobiology, Department of Medicine, Università Campus Bio-Medico di Roma, Via Alvaro del Portillo 200, 00128 Rome, Italy

20 Department of Neuroscience and Sensory Organs, Neurology Unit, Maggiore Policlinico Hospital Foundation IRCCS Ca' Granda, Milan, Italy

21 Department of Neurology and Psychiatry, Sapienza University of Rome, Rome, Italy

22 Department of Neurology, Avezzano Hospital, University of L'Aquila, L'Aquila, Italy

23 Stroke Unit and Division of Cardiovascular Medicine, University of Perugia, Perugia, Italy

24 Stroke Unit Legnano Hospital ASST Ovest Milanese, Legnano, Italy

25 IRCCS Foundation C. Mondino Neurological Institute, Pavia, Italy

26 Department of Neurosurgery, Niguarda Cà Granda Hospital, Milan, Italy

27 Laboratory of Clinical Investigations, Fondazione IRCCS Istituto Neurologico Carlo Besta, Milan, Italy

28 Neonatology Unit and Prenatal Diagnosis (P.P.), Medical Genetic Unit, Ospedale S. Maria della Misericordia, Perugia, Italy

29 Inserm UMR-S1161, Génétique et Physiopathologie des Maladies Cérébro-vasculaires, Université Paris Diderot, Sorbonne Paris Cité, Paris, France

30 Department of Neurosurgery, Charite Universitätsmedizin, Berlin, Germany 